\title{
Half-Time SPECT Myocardial Perfusion Imaging with Attenuation Correction
}

\author{
Iftikhar Ali ${ }^{1}$, Terrence D. Ruddy ${ }^{1,2}$, Abdulaziz Almgrahi ${ }^{1}$, Frank G. Anstett ${ }^{3}$, and R. Glenn Wells ${ }^{1,2}$ \\ ${ }^{1}$ Division of Cardiology, University of Ottawa Heart Institute, Ottawa, Ontario, Canada; ${ }^{2}$ Division of Nuclear Medicine, The Ottawa \\ Hospitals, University of Ottawa, Ottawa, Ontario, Canada; and ${ }^{3}$ GE Healthcare, Waukesha, Wisconsin
}

Reducing acquisition time may improve patient throughput, increase camera efficiency, and reduce costs; reducing acquisition time also increases image noise. Newly available software controls the effects of noise by maximum a posteriori reconstruction while maintaining resolution with resolution-recovery methods. This study compares half-time (HT) gated myocardial SPECT images processed with ordered-subset expectation maximization with resolution recovery (OSEM-RR) (with and without CT-based attenuation correction $[\mathrm{AC}]$ ) with full-time (FT) images obtained with a standard clinical protocol and reconstructed with filtered backprojection (FBP) and OSEM (with and without AC). Methods: A total of 212 patients (mean age, $57 \mathrm{y}$; age range, 27-86 y) underwent 1-d rest/stress $99 \mathrm{mTc}$-tetrofosmin gated SPECT. FT (12.5 min, both rest and stress) and HT (rest, $7.5 \mathrm{~min}$; stress, $6.0 \mathrm{~min}$ ) images were acquired with low-dose CT for AC in 112 patients. HT acquisitions were processed with OSEM-RR (with and without $A C$ ) using software, and FT acquisitions were processed with FBP and OSEM (with and without AC). In another 100 patients, test-retest repeatability was assessed using 2 sets of FT images (FBP reconstruction) that were acquired one immediately after the other. Radiologists unaware of the acquisition and reconstruction protocols visually assessed all reconstructed images for summed stress, summed rest, and summed difference scores and regional wall motion using a 17-segment model. Automated analysis on gated SPECT was used to determine left ventricular volumes, ejection fraction, and dilation (end-diastolic volume, end-systolic volume, left ventricular ejection fraction, and transient ischemic dilation [TID]). A clinical diagnosis was also determined. Results: All measurements resulted in significant correlations $(P<0.01)$ between the HT and FT images. The only significant difference in mean values was for OSEM-RR plus AC; this method led to an increase in TID by $4 \%$ over FT imaging. The concordance in the clinical diagnosis for HT versus FT was 106 to $112(\kappa=0.88)$ for no AC and 102 to $106(\kappa=0.91)$ for $A C$, similar to the repeatability of FT versus FT $(98 / 100, \kappa=0.95)$. Conclusion: HT images processed with the new algorithm provided a clinical diagnosis in concordance with that from FT images in 95\% (no AC) to $96 \%$ (AC) of cases. This concordance is similar to the test-retest repeatability of FT imaging.

Key Words: SPECT; myocardial perfusion imaging; resolution recovery; attenuation correction

J Nucl Med 2009; 50:554-562

DOI: 10.2967/jnumed.108.058362

Received Sep. 22, 2008; revision accepted Dec. 8, 2008.

For correspondence or reprints contact: R. Glenn Wells, Division of Cardiology Cardiac Imaging, First Floor, University of Ottawa Heart Institute, 40 Ruskin St., Ottawa, ON, K1Y 4W7, Canada.

E-mail: gwells@ottawaheart.ca

COPYRIGHT @ 2009 by the Society of Nuclear Medicine, Inc.
C oronary heart disease is a leading cause of death in the United States and the most prevalent form of cardiovascular disease, affecting 16 million people. Gated SPECT cardiac perfusion imaging plays a fundamental role in prediction, diagnosis, prognosis, and the selection of appropriate treatment for patients with suspected or known ischemic heart disease $(1,2)$.

In SPECT, there is a trade-off between image-acquisition time and noise levels. A shorter acquisition time would reduce the time during which the patient is required to lie still and reduce the likelihood of patient motion artifacts. A shorter scan time might also improve camera use and reduce the cost of the test. There was some success in reducing scan times with the introduction of multihead $\gamma$-cameras in the 1990s, which increased the detector surface area and allowed simultaneous acquisition of multiple-projection views. Other groups have suggested that diagnostic accuracy could be maintained with noisier studies (3) or studies with reduced spatial resolution (4), but these approaches had limited acceptance. More recently, there have been exciting advances in image processing and reconstruction algorithms that improve the image quality of count-poor studies.

The new algorithms incorporate noise regularization and resolution recovery (RR). To compensate for the increased noise in the shorter-duration acquisitions, some form of statistically based noise suppression, based on prior knowledge of the type of activity distribution, is applied to the data during image reconstruction. This form of noise suppression, like low-pass filtering, tends to reduce the spatial resolution of the image. To compensate for these effects, the algorithms also include distance-dependent RR. The resolution of $\gamma$ camera images decreases as the source increases its distance from the collimator surface. By modeling the point-spread function (PSF) and other sources of image blurring inside the image-creation process, it is possible to compensate for these effects and improve the image quality (5). The PSF can be integrated into reconstruction as part of an iterative algorithm. The PSF has 4 components: septal penetration, septal scatter, intrinsic detector response, and, most important, geometric response function (GRF) (6). The GRF component is defined as the normalized 2-dimensional Fourier transform of the point-source image formed in a plane behind the 
collimator by photons that are accepted geometrically through the collimator holes. It is often assumed that GRF is spatially invariant in planes parallel to the face of the collimator and that the intrinsic response is spatially invariant in the detection plane. However, a more accurate description of specific collimators can be obtained by Monte Carlo simulation or experimental measurements of collimator geometry $(7,8)$.

Our objective in this study was to compare gated myocardial perfusion images obtained using half-time (HT) acquisition and processed using one of the new RR reconstruction algorithms with those obtained using a standard clinical protocol and reconstructed using filtered backprojection (FBP) or ordered-subset expectation maximization (OSEM) in a patient population of intermediate-to-high pretest probability of coronary artery diseases (CAD).

\section{MATERIALS AND METHODS}

\section{HT Reconstruction Algorithm}

The Evolution for Cardiac software package (GE Healthcare) is a new reconstruction algorithm that has recently become available for clinical cardiac imaging. This algorithm incorporates RR and maximum a posteriori (MAP) noise regularization, permitting cardiac SPECT images to be acquired in approximately half of the time (HT acquisition) needed for reconstruction with the standard OSEM algorithm $(9,10)$. The PSF is stored as a look-up table, and the radial distance of the detector is recorded as part of the projection data. Equipment-related information for a variety of different collimators is stored in an additional look-up table that is part of the reconstruction package; acquisition parameters are retrieved from all projection data. The RR process can produce noise hot spots that detrimentally affect image quality. The MAP algorithm is used to suppress the formation of hot spots. It is a 1-step late implementation using a Green's prior with parameters that have been tuned for each clinical protocol and for gated and attenuation-corrected images (11). Attenuation correction (AC) with Evolution for Cardiac also includes scatter correction based on a dual-energy window. A median root prior is used in the last iteration of the reconstruction to preserve edge sharpness (12). This scheme has been found to give an image of equal or superior quality on limited clinical studies and on physical phantom data (13-20).

\section{Patient Population}

We prospectively evaluated 212 patients from July 2007 through March 2008 at the Nuclear Cardiology Diagnostic Imaging laboratory, University of Ottawa Heart Institute, Ottawa, Canada. Patients were selected from those who were scheduled to have routine rest/stress myocardial perfusion SPECT for suspected or known CAD. A total of 112 patients underwent full-time (FT) and HT acquisitions. Another 100 patients underwent 2 FT acquisitions to evaluate test-retest reproducibility. The patients had a mean age of $57 \mathrm{y}$ (age range, 27-86 y), and their clinical characteristics are provided in Table 1 . The test-retest group had more males and a higher pretest likelihood of CAD. However, most cases in both groups were of moderate risk $(10 \%-90 \%)$. The study was approved by the University of Ottawa's Human Ethics Review Board, and patients gave written consent before enrollment.

\section{Radiopharmaceutical Injection and Image Acquisition \\ ${ }^{99 m}$ Tc-tetrofosmin (Myoview; GE Healthcare) was used in all the studies. The radiopharmaceutical doses were administered,}

\begin{tabular}{|c|c|c|c|}
\hline Patient characteristic & FT-HT $(n=112)$ & FT-FT $(n=100)$ & $P$ \\
\hline Male & $67(60 \%)$ & 74 (74\%) & 0.03 \\
\hline Age $(y)$ & & & NS \\
\hline Mean & 58 & 56 & \\
\hline Range & $32-86$ & $27-80$ & \\
\hline Body mass index (mean $\pm S D$ ) & $28.3 \pm 4.4$ & $28.3 \pm 4.0$ & NS \\
\hline Hypertension & $74(66 \%)$ & $58(58 \%)$ & NS \\
\hline Dyslipidemia & $62(55 \%)$ & $56(56 \%)$ & NS \\
\hline Smoking (current and previous) & $59(53 \%)$ & $54(54 \%)$ & NS \\
\hline Diabetes & $18(16 \%)$ & $14(14 \%)$ & NS \\
\hline Family history of premature CAD & $46(41 \%)$ & $41(41 \%)$ & NS \\
\hline \multicolumn{4}{|l|}{ Previous event } \\
\hline Myocardial infarction & $23(21 \%)$ & $20(20 \%)$ & NS \\
\hline Percutaneous coronary intervention & $19(17 \%)$ & $25(25 \%)$ & NS \\
\hline Coronary artery bypass graft & $10(9 \%)$ & $8(8 \%)$ & NS \\
\hline \multicolumn{4}{|l|}{ Pretest likelihood of CAD (combined } \\
\hline \multicolumn{4}{|l|}{ Diamond/Forrester) } \\
\hline Low risk $(<10 \%)$ & $42(38 \%)$ & $20(20 \%)$ & 0.004 \\
\hline Moderate risk $(10 \%-90 \%)$ & $63(56 \%)$ & $73(73 \%)$ & 0.009 \\
\hline High risk $(>90 \%)$ & $7(6 \%)$ & $7(7 \%)$ & NS \\
\hline \multicolumn{4}{|l|}{ Stress test } \\
\hline Exercise & $53(47 \%)$ & $56(56 \%)$ & NS \\
\hline Dipyridamole & 59 (53\%) & $44(44 \%)$ & NS \\
\hline
\end{tabular}


and images were acquired according to the guidelines of the American Society of Nuclear Cardiology (ASNC) (21). A 1-d rest and stress imaging protocol was used in all patients. For rest images, the injected dose of ${ }^{99 \mathrm{~m}} \mathrm{Tc}$-tetrofosmin was, on average, $344 \mathrm{MBq}(9.3 \mathrm{mCi})$, with a range of $286-445 \mathrm{MBq}(7.7-12 \mathrm{mCi})$. For the stress images, the average injected dose was $1,053 \mathrm{MBq}$ (28.5 mCi), with a range of 930-1,281 MBq (25.1-34.6 mCi). All rest images were acquired at $45 \mathrm{~min}$ after tetrofosmin injection, and all stress images were acquired between 30 and 60 min after tetrofosmin injection.

\section{Exercise and Dipyridamole Stressing Protocols}

Patients were advised, at the discretion of their referring physician, to stop taking nitrates, calcium antagonists, and $\beta$-blockers at the time of the myocardial perfusion study. They were also asked to refrain from ingesting caffeine-containing foods and beverages, drugs, and dipyridamole for $24 \mathrm{~h}$ before their study.

Unless contraindicated or at the request of the referring physician, all patients underwent a symptom-limited Bruce protocol treadmill exercise test. Targeted heart rate was $85 \%$ of maximum age-adjusted predicted heart rate (22). At peak stress, the radiotracer was injected intravenously, and exercise was continued for an additional $60 \mathrm{~s}$. The patients who were unable to achieve the targeted heart rate or exercise were stressed pharmacologically. Dipyridamole $(0.142 \mathrm{mg} / \mathrm{kg} / \mathrm{min})$ was infused intravenously for $5 \mathrm{~min}$, and ${ }^{99 \mathrm{~m}} \mathrm{Tc}$-tetrofosmin was injected at $2 \mathrm{~min}$ after infusion completion (7 min into the study). Aminophyline (100-200 mg) was given intravenously $2 \mathrm{~min}$ after injection of the radiotracer.

\section{Data Acquisition and Reconstruction}

All studies were completed with a $90^{\circ}$-angled dual-head integrated SPECT/CT camera (Infinia Hawkeye 4; GE Healthcare) using standard (FT) and short (HT) protocols. All images were acquired with a low-energy high-resolution collimator using a $15 \%$ energy window centered on the $140-\mathrm{keV}$ photopeak of ${ }^{99 \mathrm{~m}} \mathrm{Tc}$, based on ASNC guidelines for cardiac ${ }^{99 \mathrm{~m}} \mathrm{Tc}$ studies (21). In the standard protocol, SPECT data were acquired for $25 \mathrm{~s}$ per projection (30 projections/head; total of $180^{\circ}$ of data) for both the rest and the stress studies. In the short protocol, data were acquired for $15 \mathrm{~s}$ per projection for the rest study and $10 \mathrm{~s}$ per projection for the stress study. Both rest and stress studies were gated at 8 frames per cardiac cycle. In one half of the 112 patients, the HT scan was acquired first, followed by the FT scan; in the other half of the patients, the scan order was reversed. A single low-dose CT scan (helical, $140 \mathrm{keV}, 1 \mathrm{~mA}$ with 1.9 pitch) was acquired at the end of both rest and stress acquisitions for AC. Vendor-supplied quality-assurance software was used to ensure the CT scan was registered with both HT and FT SPECT images. Projections and reconstructed matrix size for all scans were $64 \times$ 64 pixels, with a pixel size of $6.8 \times 6.8 \mathrm{~mm}$. Reconstruction was done on a Xeleris 2.0 (GE Healthcare) workstation running on xw6200 hardware (Hewlett Packard). For the 100 patients undergoing the test-retest portion of the study, 2 standard SPECT scans were obtained, one immediately after the other and with the same acquisition parameters as described above.

All FT scans were reconstructed with both FBP and OSEM. Both gated and ungated images were used for evaluation. The FBP images were created with a 10th-order Butterworth filter, using different cutoff frequencies for the ungated $(0.4$ cycles $/ \mathrm{cm})$ and gated $(0.35 \mathrm{cycles} / \mathrm{cm})$ data. The OSEM iterative reconstruction (2 iterations, 10 subsets) was done both with CT-based AC (IRAC) and without CT-based AC (IRNC). The OSEM images were also filtered after reconstruction with a 10th-order Butterworth filter: ungated $(0.4$ cycles $/ \mathrm{cm})$ and gated $(0.35$ cycles $/ \mathrm{cm})$.

All HT scans were reconstructed with Evolution for Cardiac using manufacturer-recommended RR and noise-reduction parameters (12 iterations, 10 subsets) both with CT-based AC (IRACRR) and without CT-based AC (IRNCRR). The factory-recommended Butterworth filters were applied to the ungated rest ( 0.4 cycles/ $\mathrm{cm})$, ungated stress $(0.52$ cycles $/ \mathrm{cm})$, and gated rest and stress $(0.4$ cycles/cm) data.

The resulting images were randomized and analyzed in a masked manner by 2 experienced American Board of Nuclear Medicine-certified nuclear medicine physicians using 4DMSPECT (Invia Medical Imaging Solutions) on a Hermes Gold workstation (Hermes Medical Solutions). Differences between the readers were resolved with consensus.

\section{Data Collection and Statistical Analysis}

The reconstructed images were assessed subjectively for image quality as poor, good, or excellent. The summed stress score (SSS) (graded from 0 [normal tracer uptake] to 4 [absent tracer]), summed rest score (SRS), and regional wall motion (graded as normal, hypokinetic, akinetic, or dyskinetic) were visually assessed using a 17-segment model. Automated analysis on gated SPECT was used to determine the end-diastolic volume (EDV), end-systolic volume (ESV), left ventricular ejection fraction (LVEF), and transient ischemic dilation (TID). Finally, a clinical diagnosis was determined from perfusion, TID, and wall motion as normal, probably normal, equivocal, probably abnormal, or abnormal. Fixed defects with normal wall motion were considered to be normal perfusion with an attenuation artifact. For this study, an SSS greater than or equal to 2 and a TID greater than 1.2 (23) were considered abnormal. A more typical cutoff value for normal SSS is less than 4 (24), but we have chosen a stricter criterion of less than 2 for this study to emphasize any minor differences in apparent perfusion.

The concordance of clinical diagnosis for the FT and HT images was evaluated using the intraclass correlation coefficient (ICC-r) and $\kappa$-coefficient. The SSS, SRS, summed difference scores (SDS), and quantitative parameters (LVEF, LVEDV, and LVESV) were compared using linear regression analysis, the ICC-r, and Bland-Altman analysis. Mean values were compared using a Bonferroni-corrected 2-tailed paired $t$ test, with a value of $P$ less than 0.05 considered significant.

\section{RESULTS}

\section{FBP Analysis}

There were no differences in subjective image quality among the FT images reconstructed with FBP and IRNC. Image quality was excellent in 111 of 112 images reconstructed with both FBP and IRNC, and the last image was adequate on IRNC. No significant difference in SSS, SRS, SDS, and wall motion were observed between FBP and IRNC (Supplemental Table 1; supplemental materials are available online only at http://jnm.snmjournals.org). Quantitative analysis of left ventricular volumes (LVEDV, LVESV, and LVEF) also showed no significant difference between FBP and IRNC. With respect to clinical diagnosis, there were 2 of $112(1.8 \%)$ discordant cases. Both cases were normal on FBP. 
The patient in the first discordant case had mild ischemia in the right coronary artery territory on IRNC, and subsequent ${ }^{82} \mathrm{Rb}$-PET findings were normal. The patient in the second discordant case also had mild ischemia in the left circumflex artery territory on IRNC but was unavailable for follow-up. This level of disagreement is similar to the discordance seen in the test-retest repeatability.

For the 100-patient test-retest portion of the study, all measures (SSS, SRS, SDS, wall motion, LVEDV, LVESV, LVEF, and TID) showed significant correlation and no significant differences in the mean values (Supplemental Table 1). Bland-Altman analysis of the LV volumes and ejection fraction showed no changes in the distribution of differences as a function of mean values. The clinical diagnosis of the 100 patients from the test-retest portion of this study showed excellent reproducibility: $\mathrm{ICCr}=0.98$ $(P=0.001)$. The patient in the first discordant case had mild-to-moderate ischemia in the inferior wall in the initial acquisition. However, in the subsequent acquisition there was scatter from the gut that led to a diagnosis of subdiaphragmatic attenuation, with normal wall motion. The patient in the second discordant case had normal perfusion in both acquisitions but had a change from normal-tomoderate global hypokinesis.

Visual Analysis

No differences in subjective image quality between FT and HT images were observed (Fig. 1). Image quality was excellent in 110 of 112 for HT images reconstructed with IRNCRR, 112 of 112 for HT images reconstructed with IRACRR, and 111 of 112 for FT images reconstructed with both IRNC and IRAC. Highly significant correlations for SSS and SRS were observed between IRNCRR and IRNC (ICCr $=0.94$ for both SSS and SRS) and between IRAC and IRACRR (ICCr $=0.96$ for both) (Supplemental Table 2). The correlation for the SDS measure was reduced

A
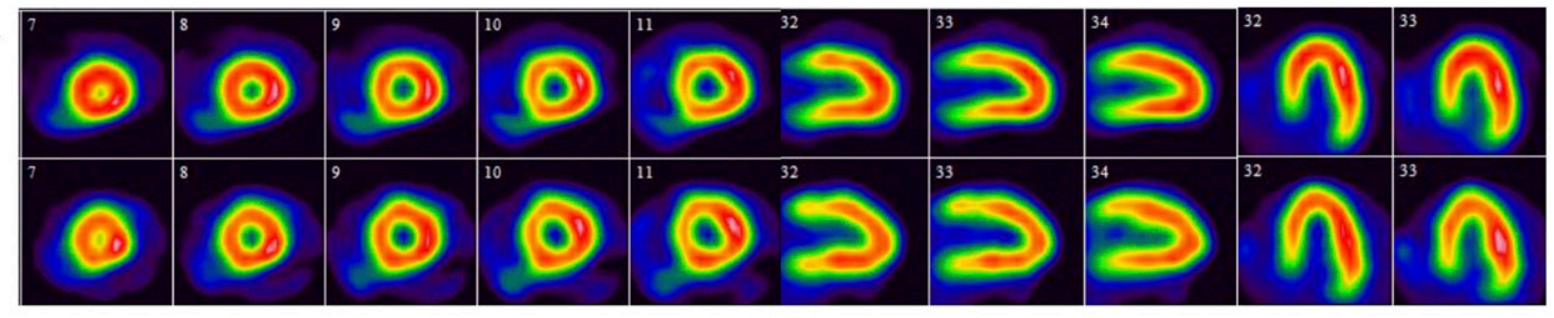

B

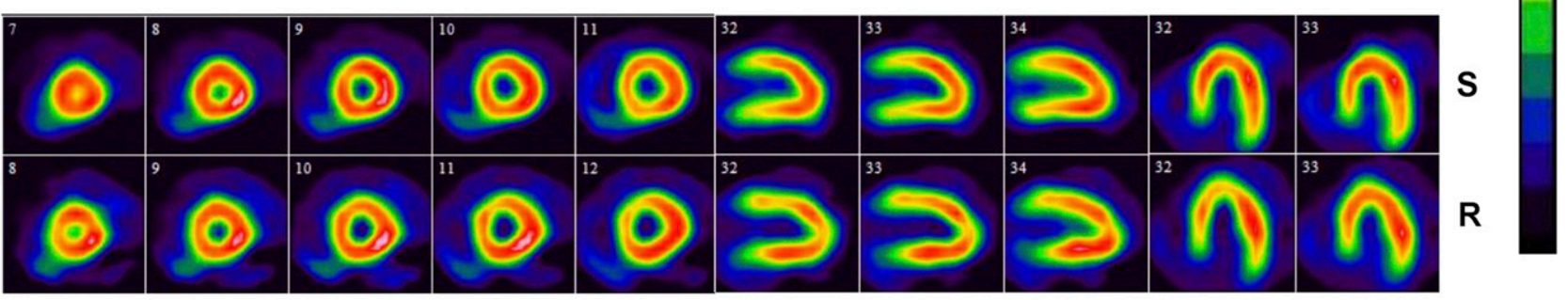

C
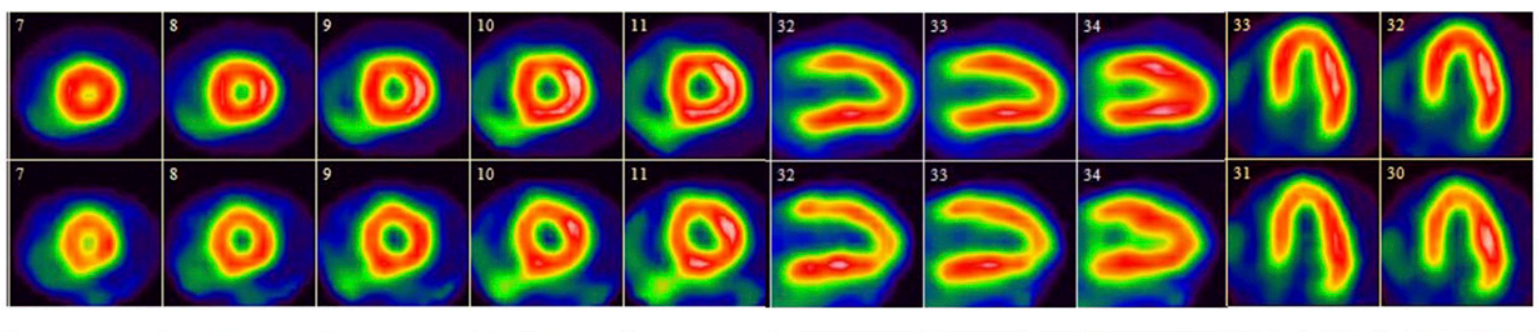

S
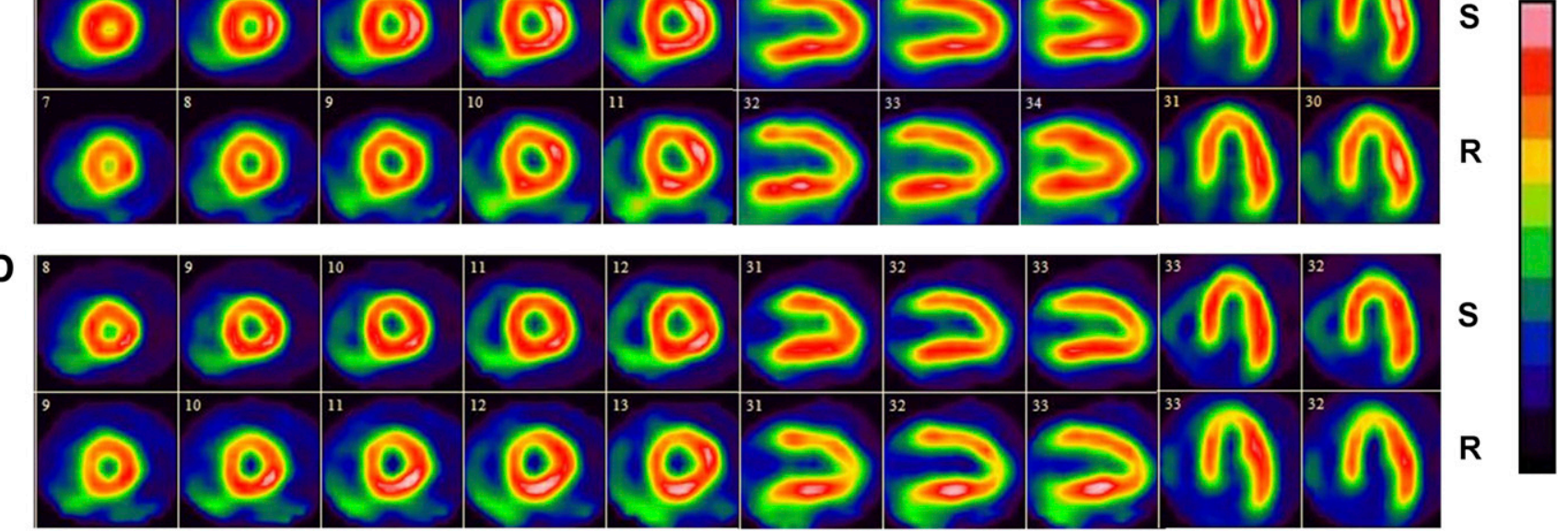

S

$\mathbf{R}$

FIGURE 1. Stress/rest ${ }^{99 m}$ Tc-tetrofosmin SPECT study. FT acquisition reconstructed with OSEM without AC (IRNC) (A), HT acquisition reconstructed with OSEM and RR without AC (IRNCRR) (B), FT acquisition reconstructed with OSEM with CT-based AC (IRAC) (C), and HT acquisition reconstructed with OSEM and RR with CT-based AC (IRACRR) (D). No difference in image quality or clinical diagnosis was observed in 95\% of cases between IRNC and IRNCRR and 96\% of cases between IRAC and IRACRR. $\mathrm{S}=$ stress; $\mathrm{R}$ = rest. 
FIGURE 2. Histogram analysis comSPECT reconstructed with (IRAC vs. IRACRR) and without (IRNC vs. IRNCRR) CT-based AC. Results for test-retest repeatability (FBP1 vs. SDS (B) are presented separately. paring $\mathrm{HT}$ and FT myocardial perfusion FBP2) are also shown. SSS (A) and
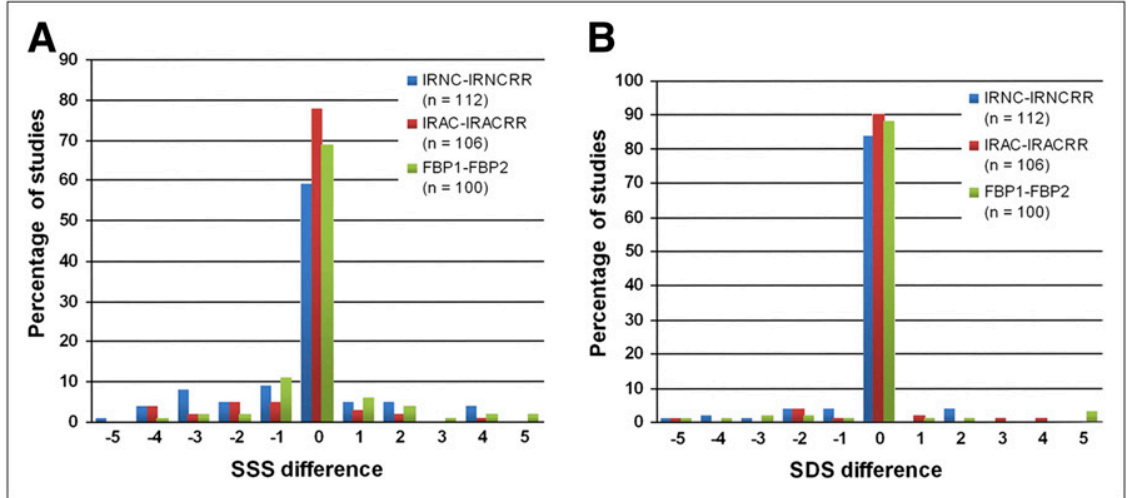

(without $\mathrm{AC}, \mathrm{ICCr}=0.55$; with $\mathrm{AC}, \mathrm{ICCr}=0.39$ ). The reduced correlation was caused by the large number of patients with an SDS of 0 and the small range of SDS differences in our patient sample (range, -5 to 4 ). The concordance in SDS remained good; the SDS differences between FT and HT images (Fig. 2) were 0 in 95 of 112 patients without $\mathrm{AC}$ and 96 of 106 patients with $\mathrm{AC}$ and were greater than or equal to 4 in only 3 of 112 patients without $\mathrm{AC}$ and 2 of 106 patients with $\mathrm{AC}$.

\section{Quantitative Analysis of LV Volume and Function}

Quantitative analyses were performed on both FT and HT gated stress acquisitions. We compared HT and FT images without (IRNCRR and IRNC) and with CT-based AC (IRACRR and IRAC) and found no significant differences in LVEDV, LVESV, and LVEF (Supplemental Table 2). LVEF determined from HT SPECT correlated well with that of FT without AC (IRNC vs. IRNCRR, ICCr $=0.90$ ) and with CT-based AC (IRACRR vs. IRAC, $\mathrm{ICCr}=0.88$ ) (Fig. 3). The Bland-Altman plot analysis of IRNCRR with IRNC and IRACRR with IRAC showed no significant differences in LVEF (Fig. 4). Similar results were seen for LVEDV and LVESV (Supplemental Table 2). The only difference observed was a small but significant $(P=0.04)$ increase in the average TID for IRACRR (TID $=0.96$ ), compared with IRAC $($ TID $=0.92)$.

\section{Clinical Diagnosis}

Clinical diagnoses obtained from IRNCRR were 95\% (106/112) concordant with IRNC, and the $\kappa$-coefficient was 0.88. Clinical diagnoses obtained from IRACRR were $96 \%$ $(102 / 106)$ concordant with IRAC, and the $\kappa$-coefficient was 0.91. The differences in SSS in the 6 discordant cases were mild; they were within the range of 2 to 4 . Frequently an SSS greater than or equal to 4 is required for a scan to be considered abnormal. In this study, we use an SSS cutoff of 2. We also used a TID value greater than or equal to 1.2 as abnormal and considered fixed defects with normal wall motion to be attenuation artifacts (for a scan without AC). The discordant clinical diagnoses (Table 2) are detailed in Table 3. CT-based AC resolved all of the differences in perfusion (cases 2-6) (Fig. 5). In case 1, the patient was claustrophobic and a CT scan could not be acquired. An
${ }^{82} \mathrm{Rb}-\mathrm{PET}$ follow-up study showed normal perfusion. Patients in cases 5 and 6 were also followed up with an electrocardiogram-gated ${ }^{82} \mathrm{Rb}$-PET scans, and both PET studies had normal perfusion and normal TID. The patient in case 7 underwent a subsequent angiogram during the course of clinical evaluation, which showed no significant stenosis supplying the inferolateral wall, the area of ischemia noted in both no-AC studies and the IRAC study. The patient in case 8 relocated and was unavailable for further follow-up.

\section{DISCUSSION}

This study compared images obtained with HT acquisition and processed with the Evolution for Cardiac reconstruction algorithm with conventional image acquisition and processing protocols using FBP and OSEM. Our results indicate that the new reconstruction algorithm with RR can reduce acquisition time and provide similar image quality and diagnostic accuracy for myocardial perfusion in terms of perfusion, wall motion, and volumes for the evaluation

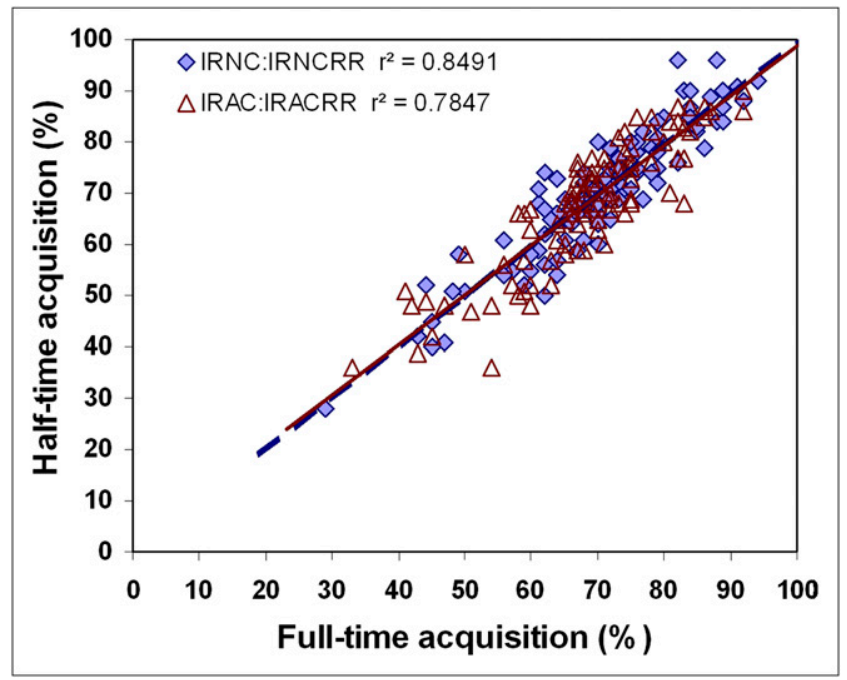

FIGURE 3. Linear regression analysis of LVEF comparing $H T$ and FT myocardial perfusion SPECT with (IRAC vs. IRACRR) and without (IRNC vs. IRNCRR) CT-based AC. 

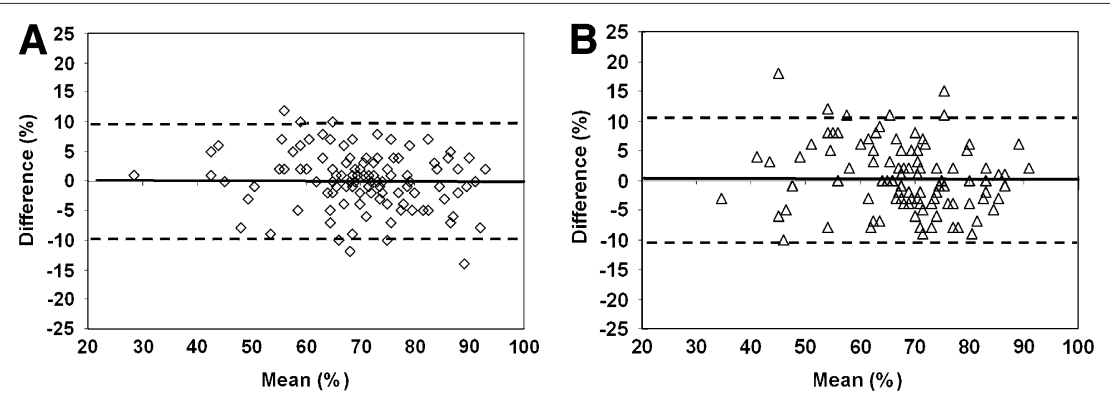

FIGURE 4. Bland-Altman analysis of LVEF comparing HT and FT myocardial perfusion SPECT without (IRNC vs. IRNCRR) (A) and with (IRAC vs. IRACRR) (B) CT-based AC. LVEF (IRNC vs. IRNCRR): mean difference, $0.1 ; 1.96$ SDs, 9.4. LVEF (IRAC vs. IRACRR): mean difference, $0.4 ; 1.96 \mathrm{SDs}, 11.0$.

of CAD. Moreover, these results are similar both with and without the application of CT-based AC. Mild discrepancies in FT-HT are similar to test-retest repeatability of FT imaging.

The depth-dependant RR approach has been adapted by various vendors in their new reconstruction algorithms with various methods of modeling components: Evolution for Cardiac $(10,13,15,20)$, Wide-Beam Reconstruction (WBR) (UltraSPECT) $(10,13,15,16)$, Astonish (Philips Medical System) $(17,18)$, and 3D Flash (Siemens Medical Solutions) (19). Our study supports and expands on the results and conclusions of other work done previously with the Evolution for Cardiac package. DePuey et al. compared 2 new reconstruction algorithms with FBP. These authors demonstrated that for cardiac perfusion SPECT, HT Evolution for Cardiac (OSEM-RR) and WBR offered stress and rest image quality and diagnostic accuracy equivalent to FT FBP $(13,15)$. DePuey et al. also demonstrated that these new methods (OSEM-RR and WBR) offered gated myocardial perfusion SPECT functional image quality with HT acquisitions equivalent to or possibly superior to that achieved with FT acquisition processed with FBP (10). In the studies by these authors, images were acquired on a Ventri $90^{\circ}$-angled dual-head scintillation camera (GE Healthcare), and we have demonstrated similar results here on an Infinia Hawkeye (GE Healthcare). DePuey et al. compared HT OSEM-RR and WBR with FT FBP using metrics of image quality, LV volumes, LVEF, and wall motion. In our study, we extended these results to clinical diagnosis, SSS, SRS, SDS, and CT-based AC. We had also compared 2 sets of FT acquisitions reconstructed with a clinically used FBP algorithm for test-retest reproducibility.

This study suggests that CT-based AC may improve concordance between HT and FT images. In those cases for which there was discordance between the IRNC and IRNCRR results, the inclusion of CT-based AC removed the differences in perfusion. A possible explanation is that the addition of AC in the algorithm improves the accuracy of the camera over RR alone and leads to greater consistency in the data and a more accurate reconstructed image. Narayanan et al. demonstrated that iterative reconstruction provides better performance than does FBP reconstruction for the overall detection of $\mathrm{CAD}$ and the localization of perfusion defects in the 3 arterial territories (25). They noted a steady improvement in defect detection as attenuation, scatter, and resolution losses were progressively compensated for in the reconstruction, suggesting that improved modeling of physical degradations can improve the quality of cardiac perfusion images. Thus, use of both resolution and attenuation compensation might be expected to yield a better result than would resolution compensation alone, although with the small number of cases in this study the differences were not shown to be significant.

A 100-patient test-retest study was performed on the same camera with the same methodology to determine the reproducibility for specific parameters (SSS, SRS, SDS, LVEDV, LVESV, LVEF, and wall motion) and clinical diagnosis through masked reading of separate studies performed one immediately after the other. This study yielded a discrepancy rate for the clinical diagnosis of $2 \%$. Although this rate is slightly lower than the discrepancy rate seen between the FT and HT images, the small test-retest variability would account for some of the discrepancy seen, making the true difference between the HT and FT images even less.

In this study, we observed a small but significant $(P=0.04)$ increase of $4 \%$ in the average TID for IRACRR, compared with IRAC. TID may be caused by an apparent thinning of the myocardial wall due to reduced uptake in the endocardium (26). TID is measured on the basis of the change in LV volume between rest and stress images. Resolution recovery has the potential to alter the spatial resolution of images and thereby influence the accuracy of edge detection and measured vol-

\section{TABLE 2. Clinical Diagnosis Without and With \\ CT-Based AC \\ HT

\begin{tabular}{lrc}
\cline { 2 - 3 } FT & Normal & Abnormal \\
No AC & & \\
Normal & 69 & 5 \\
Abnormal & 1 & 37 \\
AC & 71 & 2 \\
Normal & 2 & 31 \\
Abnormal & &
\end{tabular}

Normal studies had normal perfusion, TID, and wall motion. Abnormal studies had SSS greater than or equal to 2 or TID greater than 1.2 or wall motion abnormalities greater than or equal to 2 . 


\begin{tabular}{|c|c|c|c|c|c|c|}
\hline \multirow[b]{2}{*}{ Case no. } & \multicolumn{3}{|c|}{ No AC (IRNC vs. IRNCRR) } & \multicolumn{3}{|c|}{ CT-based AC (IRAC vs. IRACRR) } \\
\hline & Perfusion (SDS) & TID & WM & Perfusion & TID & WM \\
\hline 1 & $\mathrm{X}(2,0)$ & & & * & & \\
\hline 2 & $X(0,3)$ & & & & & \\
\hline 3 & $X(0,4)$ & & & & & \\
\hline 4 & $X(0,2)$ & & & & & \\
\hline 5 & $X(0,2)$ & & & & $X(1.03,1.25)$ & \\
\hline 6 & $X(0,1)$ & $X(1.03,1.3)$ & & & $X(1.04,1.27)$ & \\
\hline 7 & & & & $\mathrm{X}$ & & \\
\hline 8 & & & & $x$ & & \\
\hline
\end{tabular}

${ }^{*} \mathrm{CT}$ was unavailable (because of patient's claustrophobia).

Differences in perfusion were mild in all cases (SDS of $\leq 4)$. In cases of discordant TID results, values of TID for 2 image sets are noted in parentheses.

umes. In light of this and the observed increase in TID, it may be necessary to redefine the reference range for TID using the HT reconstruction algorithm.
A disadvantage of the new reconstruction algorithm is that there is a significant increase in image reconstruction time with RR (11 min for each IRNCRR and IRACRR),

A
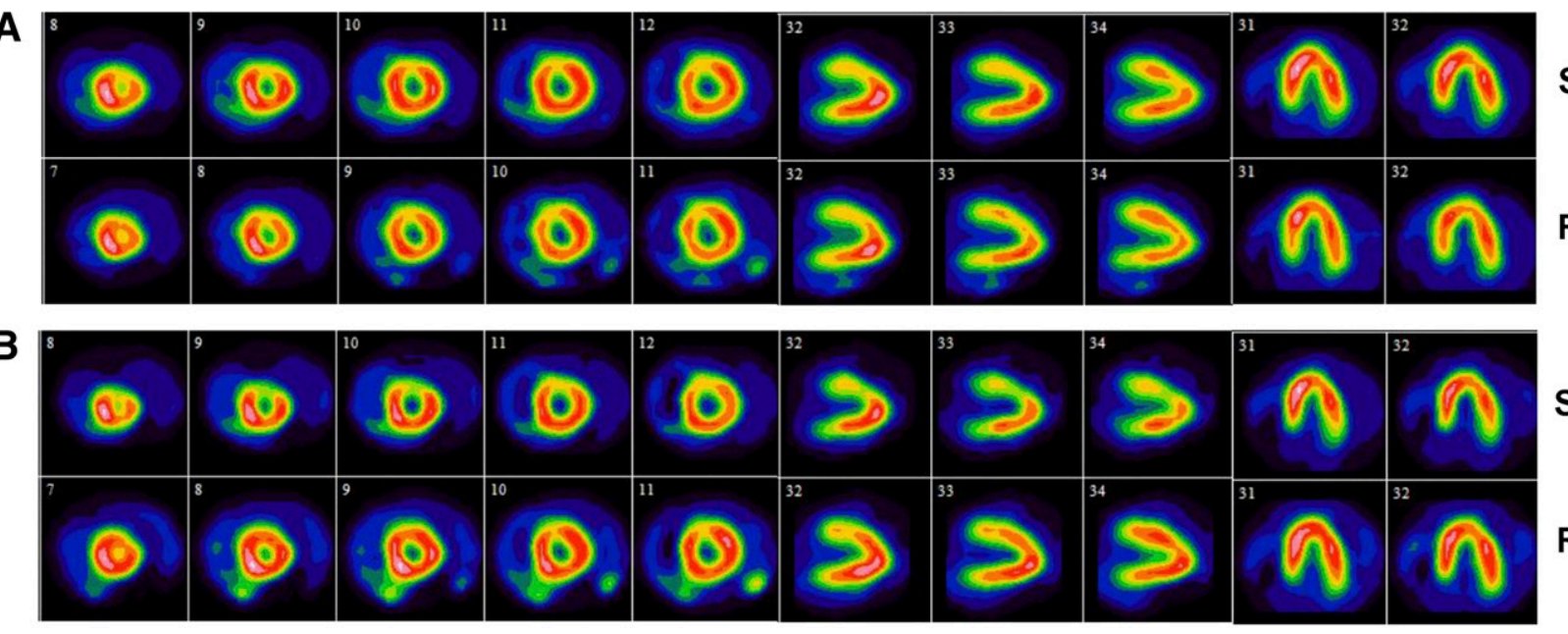

C
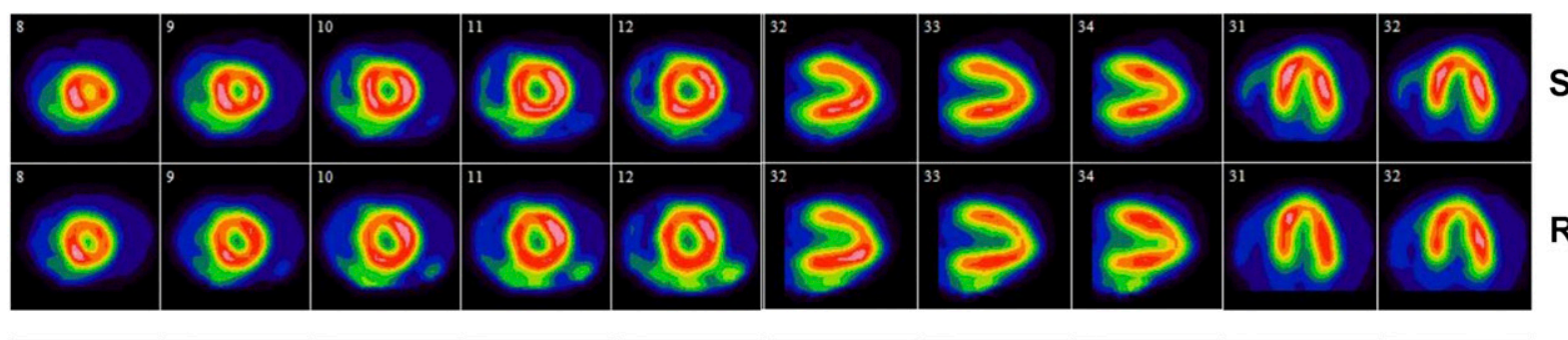

D
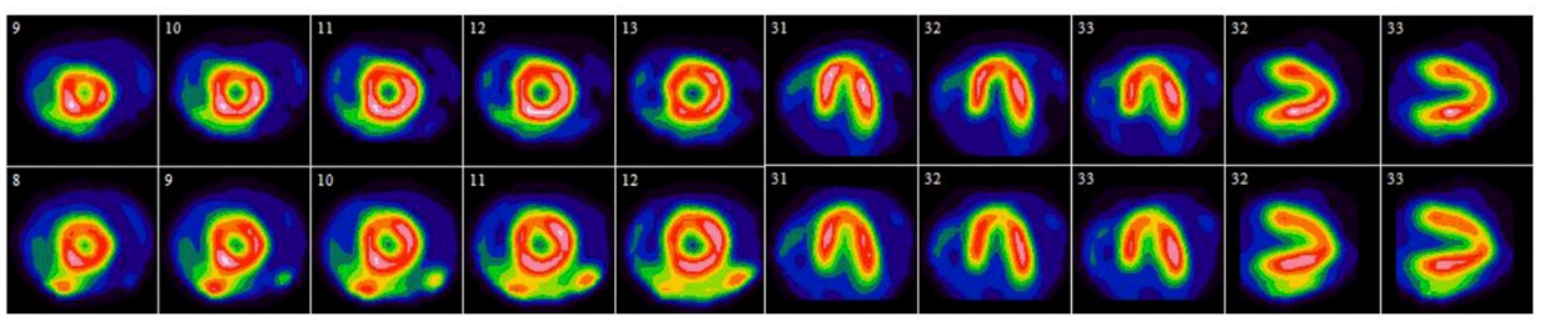

FIGURE 5. Stress/rest $99 \mathrm{~m}$ Tc-tetrofosmin SPECT study. FT acquisition reconstructed with OSEM without AC (IRNC) shows breast attenuation in anterior wall $(A), H T$ acquisition reconstructed with OSEM and RR without $A C$ (IRNCRR) shows reversible defect in anterior wall (B), FT acquisition reconstructed with OSEM with AC (IRAC) shows normal perfusion (C), and HT acquisition reconstructed with OSEM and RR with CT-based AC (IRACRR) also shows normal perfusion (D). $S=$ stress tetrofosmin study; $R$ = rest tetrofosmin study. 
compared with standard clinical reconstruction (1-2 min for FBP and 2-3 min for IRNC). The increase in processing time might have the potential to affect workflow, offsetting the reduced acquisition time. However, for the purposes of image-quality assurance, the projection data are immediately available. In addition, the total reconstruction time is still considerably less than camera time required for each patient when the time for patient positioning and camera set-up are included. Thus, reconstructed images are completed before the next patient's projection data become available and no backlog in processing should occur.

\section{Study Limitations}

The RR and noise reduction described in this study for HT acquisition are modeled specifically for each camera and collimator; thus, the result of 1 camera cannot be completely generalized to other types of cameras and collimators. The result of the study described in this report is validated only for the Infinia Hawkeye 4 SPECT/CT dual-head camera. Therefore, care should be taken in extending these results to other systems, and further validation may be necessary.

The filters used for the FT images were different from those recommended for the HT images. Incorporating RR and MAP priors into the reconstruction alters the noise and resolution properties of the resultant images, making it unlikely that the optimal filters for the new algorithm will coincide with those currently used as optimal for our clinical (FT) images. In this study, we chose to use the manufacturerrecommended postfiltering parameters. These are expected to be a reasonable choice for filtering but may not represent the optimal choice for our patient population. The differences in filtering combined with RR may alter the definition of the myocardial wall and could, therefore, alter the automatic computation of the valve plane or ventricular volume, for example. The filters could possibly be adjusted to improve further the already high concordance between the FT and HT images. In addition, some studies have shown that imaging early after stress $(<30 \mathrm{~min})$ can result in transient wall motion abnormalities (27). This effect may have contributed to differences seen in some cases because of the serial nature of our imaging protocol.

Differences were noted between some FT and HT images for which follow-up was not available. This was true for images both with and without AC, although there was a trend toward a smaller number of discrepancies with the inclusion of AC. Because of the lack of an independent standard, it remains uncertain which of the approaches yields a more accurate result in this small number of cases. Additionally, the total number of discrepancies between the HT and FT images is small, making it difficult to draw any meaningful conclusion as to whether the images from one approach are superior to those of the other. Further investigation with an independent standard such as PET would be necessary to settle this question, and the results are unlikely to be clinically meaningful as the number of studies in question is $4 \%$ or fewer.

\section{Clinical Relevance}

The advantages of reducing acquisition time by $50 \%$ are considerable and include improvement in image quality through reduced patient motion, improvement in patient experience with less discomfort and anxiety, improvement in patient throughput, and, finally, possible increase in camera efficiency. Alternatively, the radiopharmaceutical dose could be halved while maintaining a FT acquisition, to decrease radiation exposure to patients and staff.

\section{CONCLUSION}

A new OSEM-based reconstruction algorithm incorporating depth-dependant RR and MAP noise suppression has been evaluated in 112 patients both with and without CTbased AC. HT images processed with the new algorithm provided a clinical diagnosis in concordance with the diagnosis from FT images processed with standard algorithms in 95\% (no AC) to $96 \%$ (with AC) of cases. The concordance of the FT-HT imaging is similar to test-retest repeatability of FT imaging.

\section{ACKNOWLEDGMENTS}

We gratefully acknowledge the technical assistance of Patti M.E. Irvine, Rene Adeny, Elaine Cooper, Brian F. Marvin, Marlie A. Poirier, and Lyanne N. Fuller. We thank GE Healthcare for providing the Evolution for Cardiac software package. This study was funded in part by the Ontario Research Fund (ORF)-Research Excellence Program. One author is supported by a research fellowship funded by the University of Ottawa, Ottawa, Canada.

\section{REFERENCES}

1. DePuey EG, Rozanski A. Using gated technetium-99m-sestamibi SPECT to characterize fixed myocardial defects as infarct or artifact. J Nucl Med. 1995; 36:952-955.

2. Thomas GS, Miyamoto MI, Morello AP, et al. Technetium 99m sestamibi myocardial perfusion imaging predicts clinical outcome in the community outpatient setting: the Nuclear Utility in the Community (NUC) Study. J Am Coll Cardiol. 2004;43:213-223.

3. Taillefer R, Primeau M, Lambert R, Levelle Y. Technetium-99m-sestamibi myocardial perfusion imaging: comparison between a short ( 8 minutes) and standard (21 minutes) data acquisition time in diagnosis of coronary artery disease [abstract]. J Nucl Med. 1992;33(suppl):855P.

4. DePuey EG, Nichols KJ, Slowikowski JS, et al. Fast stress and rest acquisitions for technetium-99m sestamibi separate day SPECT. J Nucl Med. 1995;36:569574.

5. Gifford HC, King MA, Wells RG, Hawkins WG, Narayanan MV, Pretorius PH. LROC analysis of detector response compensation in SPECT. IEEE Trans Med Imaging. 2000;19:463-473.

6. Frey EC, Tsui BMW. Collimator-detector response compensation in SPECT. In: Zaidi H, ed. Quantitative Analysis in Nuclear Medicine Imaging. Berlin, Germany: Springer; 2005.

7. Metz CE, Atkins FB, Beck RN. The geometric transfer function component for scintillation camera collimators with straight parallel holes. Phys Med Biol. 1980;25:1059-1070.

8. Tsui BMW, Gullberg GT. The geometric transfer-function for cone and fan beam collimators. Phys Med Biol. 1990;35:81-93.

9. Tsui BMW, Frey EC, Zhao X, Lalush DS, Johnston RE, McCartney WH. The importance and implementation of accurate 3D compensation methods for quantitative SPECT. Phys Med Biol. 1994;39:509-530. 
10. DePuey G, Gadiraju R, Clark J, Thompson L, Anstett F, Schwartz SC. Ordered subset expectation maximization and wide beam reconstruction "half-time" gated myocardial perfusion SPECT functional imaging: a comparison to "fulltime" filtered backprojection. J Nucl Cardiol. 2008;15:547-563.

11. Green PJ. Bayesian reconstruction from emission tomography data using a modified EM algorithm. IEEE Trans Med Imaging. 1990;9:84-93.

12. Alenius S, Ruotsalainen U. Bayesian image reconstruction for emission tomography based on median root prior. Eur J Nucl Med. 1997;24:258-265.

13. DePuey G, Gadiraju R, Anstett F, et al. Half-time gated myocardial perfusion SPECT processed with both OSEM with resolution recovery and wide beam reconstruction: clinical performance compared to full-time filtered backprojection. In: Proceedings of the ICNC meeting; May 2, 2007; Prague, Czech Republic. Abstract 472.

14. Belhocine T, Gambhir S, Brenner R, Peretz A, Driedger A, Urbain JL. Half-time resolution recovery package for SPECT-CT MPI: a pilot study [abstract]. J Nucl Med. 2007;48(suppl):234P.

15. DePuey G, Gadiraju R, Anstett F. OSEM and WBR half-time gated myocardial perfusion SPECT: a comparison to filtered backprojection [abstract]. $\mathrm{J} \mathrm{Nucl}$ Med. 2007;48(suppl):237P.

16. Borges-Neto S, Pagnanelli RA, Shaw LK, et al. Clinical results of a novel wide beam reconstruction method for shortening scan time of Tc-99m cardiac SPECT perfusion studies. J Nucl Cardiol. 2007;14:555-565.

17. Heller GV, McGhie AI, Philips RD, et al. Multi-center evaluation of a new reconstruction method with depth dependent collimator resolution and scatter compensation applied to half-time small field of view SPECT MPI. Paper presented at: ASNC 2007 meeting; September 6-9; San Diego, California.

18. Bateman TM, Heller GV, McGhie AI. Application of simultaneous Gd-153 line source attenuation correction to half-time SPECT acquisitions: multi-center clinical evaluation. Paper presented at: ASNC 2007 meeting; September 6-9; San Diego, California.
19. Kritzman JN, Cahill JM, Ficaro EP, Corbett JR. Quantitative comparison of standard and reduced acquisition time attenuation corrected and non-corrected myocardial perfusion images: a phantom study utilizing a 3D iterative reconstruction [abstract]. J Nucl Cardiol. 2007;14(suppl 1):S107-S108.

20. Valenta I, Treyer V, Husmann L, et al. New reconstruction algorithm allows shortened acquisition time for myocardial perfusion SPECT [abstract]. $J$ Nucl Med. 2008;49(suppl 1):72P.

21. Hansen CL, Goldstein RA, Akinboboye OO, et al. Myocardial perfusion and function: single photon emission computed tomography. J Nucl Cardiol. 2007; 14:e39-e60.

22. Henzlova MJ, Cerqueira MD, Mahmarian JJ, Yao SS. Stress protocols and tracers. J Nucl Cardiol. 2006;13:e80-e90.

23. Abidov A, Bax JJ, Hayes SW, et al. Transient ischemic dilation ratio of the left ventricle is a significant predictor of future cardiac events in patients with otherwise normal myocardial perfusion SPECT. J Am Col Cardiol. 2003;42: 1818-1825.

24. Iskandrian AE. Risk assessment of stable patients (panel III) [abstract]. $J$ Nucl Cardiol. 1999;6:93.

25. Narayanan MV, King MA, Pretorius PH, et al. Human-observer receiveroperating-characteristic evaluation of attenuation, scatter, and resolution compensation strategies for ${ }^{99 \mathrm{~m}} \mathrm{Tc}$ myocardial perfusion imaging. J Nucl Med. 2003;44:1725-1734.

26. Iskandrian AS, Heo J, Nguyen T, Lyons E, Paugh E. Left ventricular dilatation and pulmonary thallium uptake after single-photon emission computer tomography using thallium-201 during adenosine-induced coronary hyperemia. Am J Cardiol. 1990;66:807-811.

27. Giorgetti A, Rossi M, Stanislao M, et al. Feasibility and diagnostic accuracy of a gated SPECT early imaging protocol: a multicenter study of the Myoview imaging optimization group. J Nucl Med. 2007;48:1670-1675. 